\title{
An Estimated General Cross Validation Function for Periodic Control Theoretic Smoothing Splines
}

\author{
M. Karasalo X. Hu and C. F. Martin
}

\begin{abstract}
In this paper, a method is developed for estimating the optimal smoothing parameter $\varepsilon$ for periodic control theoretic smoothing splines. The procedure is based on general cross validation $(G C V)$ and requires no a priori information about the underlying curve or level of noise in the measurements. The optimal $\varepsilon$ is the minimizer of a $G C V$ cost function, which is derived based on a discretization of the $L_{2}$ smoothing problem for periodic control theoretic smoothing splines.
\end{abstract}

Keywords: general cross validation, optimal smoothing, influence matrix, periodic control theoretic smoothing splines

\section{E.1 Introduction}

In this paper, we consider the problem of estimating representations of objects or contours using a type of continuous closed curves, the periodic control theoretic smoothing splines. The splines are retrieved from noisy measurements of an unknown, underlying contour. Intended applications include mapping, identification and path planning for autonomous agents. The focus of this paper is the issue of the level of smoothing, determined by the magnitude of the so called smoothing parameter $\varepsilon$.

It is well known that an interpolating spline generated from noisy measurements yields a poor estimate of the underlying curve, as the spline will pass through every measurement point. An interpolating spline may be regarded as a smoothing spline with $\varepsilon=\infty$, while a periodic smoothing spline with $\varepsilon \rightarrow 0$ approaches a circle. The theory of regular smoothing splines, and the particular issue of choice of smoothing parameter, is treated in [13-15]. Control theoretic smoothing splines have been studied in [1-7] and it has been shown in [5] that such splines, where the curve is found through minimizing a cost function, act as filters and are better suited for noisy measurements. A thorough treatment of control theoretic smoothing splines is provided in the book [8].

The particular type of periodic control theoretic smoothing spline explored in this paper has been previously presented in [9-11]. These publications cover error convergence properties for a recursive formulation of the smoothing spline problem. Experimental results indicate that the convergence is fairly robust with respect to the choice of smoothing parameter $\varepsilon$, but a formal 
method of finding the optimal value of $\varepsilon$ has so far been lacking in our work. In this paper, a method is developed for determining the appropriate level of smoothing, assuming that the shape of the underlying contour, as well as the level of noise in the measurements, is unknown. We propose an estimate of the general cross validation $(G C V)$ function, based on the estimated influence matrix for the smoothing spline problem. A general expression for the influence matrix based on Bernoulli polynomials is derived in [12]. However, this expression is computationally heavy. In [16-18], the trace of the influence matrix is estimated and an estimate of the $G C V$ function itself is obtained by Taylor expansion. $[19,20]$ and others use singular value decomposition to estimate the $G C V$ function. In this paper, on the other hand, we derive an estimate of the influence matrix and $G C V$ function, based directly upon a discretization of the underlying spline problem. The method is straightforward and easy to implement, and the accuracy can be chosen arbitrarily by adjusting the number of discretization points.

The paper is organized as follows. The contour estimation problem is formally stated in Section E.2. In Section E.3 we derive a discretization of the optimization problem. The $G C V$ approach to optimal smoothing is reviewed in Section E.4 and the specific estimated GCV function is introduced. This constitutes the main contribution of the paper. Finally, simulation results are presented in Section E.5 and conclusions are drawn in Section E.6.

\section{E.2 Preliminaries}

Consider the problem of reconstructing continuous, closed curves in $\mathbb{R}^{2}$ from noisy and sparse measurement data. This problem arises for instance in mapping applications for mobile robots. A formal problem statement follows.

Given a data set $D=\left\{\left(t_{i}, z_{i}\right): i=1, \ldots, N\right\}$, where $t_{i} \in[0,2 \pi]$ is the polar coordinate angle and $z_{i}$ is the radius in polar coordinates. If $z_{i}=r\left(t_{i}\right)+\xi_{i}, \xi_{i} \in N\left(0, \sigma^{2}\right)$, where $r\left(t_{i}\right)$ are samples from a closed smooth curve, how to find the function $r(t)$ that best represents the underlying curve, with respect to smoothness and closeness to measurement data?

The solution is found by solving the following polar second derivative $L_{2}$ smoothing problem:

Problem E.2.1

$$
\begin{aligned}
& \underset{u(t) \in L_{2}[0, T]}{\operatorname{minimize}} \quad J(u, r)=\int_{0}^{2 \pi} u(t)^{2} d t+\frac{\varepsilon^{2}}{N} \sum_{i=1}^{N}\left(r\left(t_{i}\right)-z_{i}\right)^{2} \\
& \text { subject to } r^{\prime \prime}(t)=u(t) \\
& r(0)=r(2 \pi) \\
& r^{\prime}(0)=r^{\prime}(2 \pi) .
\end{aligned}
$$

The solution of Problem E.2.1 is the optimal compromise between smoothness of the output curve, due to the integral term in $J(u, r)$, and faithfulness to the data set, due to the summation term. The magnitude of the smoothing parameter $\varepsilon>0$ determines how much credibility is given to measurement data. A large value brings the spline close to the data points, while a small value yields a smoother spline and thus more filtering. The main topic of this paper is how to determine the best choice of $\varepsilon$, based on the discretization of Problem E.2.1 reviewed in the next section.

\section{E.3 Discretization}

Using a proper choice of approximation formulas, Problem E.2.1 is reduced to an unconstrained quadratic programming problem $(Q P)$, suitable for numeric implementation. With this particular choice of discretization the periodic boundary condition is embedded in the $Q P$, facilitating the analysis of convexity and solvability for the problem. 
Let the vectors $\hat{r}=\left\{\hat{r}_{m}\right\}$ and $\hat{u}=\left\{\hat{u}_{m}\right\}$ be the discretizations of the spline $r(t)$ and control $u(t)$, and let $\hat{t}=\left\{\hat{t}_{m}\right\}$ be the corresponding discretization of $t$. Here $m=1, \ldots, M$ and the sampling rate $h$ is defined so that $(M+1) h=2 \pi$. We emphasize that $\left(\hat{t}_{m}, \hat{r}_{m}\right)$ are equidistant samples from the spline $r(t)$ while $\left(t_{i}, z_{i}\right), i=1, \ldots, N$ are noisy measurement data from the true curve. Let $z=\left\{z_{i}\right\}$ denote the vector of radius measurements. Note that when the continuous function $r(t)$ is expressed in discretized form as a vector pair $(\hat{t}, \hat{r})$, the periodicity constraint translates to $\left(\hat{t}_{1}, \hat{r}_{1}\right)=\left(\hat{t}_{M+1}, \hat{r}_{M+1}\right)$, where $M+1$ indicates the point after the last point of the vector. $\hat{u}_{m}$ can be approximately expressed as functions of $\hat{r}$, using numerical differentiation:

$$
\hat{u}_{m}=\hat{r}_{m}^{\prime \prime}=\left(1 / h^{2}\right)\left(\hat{r}_{m-1}-2 \hat{r}_{m}+\hat{r}_{m+1}\right) .
$$

Construct the matrix $\Phi \in \mathbb{R}^{M \times M}$ :

$$
\begin{aligned}
\Phi_{m, m-1} & =\Phi_{m, m+1}=1 \\
\Phi_{1, M} & =\Phi_{M, 1}=1 \\
\Phi_{m, m} & =-2 \\
\Phi_{j, l} & =0 \text { otherwise. }
\end{aligned}
$$

Then $\hat{u}=\frac{1}{h^{2}} \Phi \hat{r}$. Note that the periodicity is implicitly expressed in $\Phi$. The discretization of the integral is

$$
\int_{0}^{2 \pi} u(t)^{2} d t \approx \frac{1}{h^{3}} \hat{r}^{T} \Phi^{T} \Phi \hat{r}
$$

For the summation term, construct the matrix $F \in \mathbb{R}^{M \times N}$ :

$$
F_{m, i}= \begin{cases}1 & \text { if } \hat{t}_{m}=t_{i}, \text { for some } m \in[1, M], i \in[1, N] \\ 0 & \text { otherwise }\end{cases}
$$

$F$ expands a vector of $N$ measurements to an $M$-vector with the measurements inserted at the corresponding sampling times and zeros elsewhere. We get

$$
\frac{\varepsilon^{2}}{N} \sum_{i=1}^{N}\left(r\left(t_{i}\right)-z_{i}\right)^{2} \approx \frac{\varepsilon^{2}}{N}\left(\hat{r}^{T} F F^{T} \hat{r}+z^{T} z-2(F z)^{T} \hat{r}\right) .
$$

Remark E.3.1 We assume that the sampling times are unique so that there is one data point for each value of $i$. In reality, $t_{i}$ may not coincide with a grid point $\hat{t}_{m}$, but with $M>>N$, choosing the grid point closest to $t_{i}$ gives a negligible error.

Finally, the discretization of Problem E.2.1 is

Problem E.3.1

$$
\begin{aligned}
\underset{\hat{r}}{\operatorname{minimize}} & \hat{J}(\hat{r})=\frac{1}{2} \hat{r}^{T} H \hat{r}+c^{T} \hat{r} \\
\text { where } H & =\frac{1}{h^{3}} \Phi^{T} \Phi+\frac{\varepsilon^{2}}{N} F F^{T} \\
c & =-\frac{\varepsilon^{2}}{N} F z .
\end{aligned}
$$

Proposition E.3.1 Define $\mathbf{F} \triangleq\left(\varepsilon^{2} / N\right) F F^{T}$. Assume that $\mathbf{F}_{m m}>0$ for at least one value of $m \in[1, M]$ (this is equivalent to having a non-empty data set). Then the $Q P(E .8)$ has a unique solution. 


\section{Proof}

If $H$ is positive definite, the $Q P(\mathrm{E} .8)$ is strictly convex and has the unique solution

$$
\hat{r}=-H^{-1} c .
$$

The matrix $\frac{1}{h^{3}} \Phi^{T} \Phi$ is by construction a symmetric positive semidefinite matrix, but not positive definite since $\operatorname{rank}(\Phi)=M-1$. The single zero eigenvalue of $\Phi$ has the eigenvector $v_{0}=[1,1, \ldots, 1]^{T}$. F is diagonal with nonnegative elements and has rank $N$. Now, any $x \in \mathbb{R}^{M}$ can be decomposed as

$$
x=v+\alpha v_{0}, \quad \alpha \in \mathbb{R}, \quad v \perp v_{0},
$$

so that

$$
x^{T} \Phi^{T} \Phi x=v^{T} \Phi^{T} \Phi v \geq 0,
$$

with equality only for $v=0$. Therefore,

$$
x^{T} H x=\frac{1}{h^{3}} v^{T} \Phi^{T} \Phi v+x^{T} \mathbf{F} x .
$$

If $x$ is nonzero, then at least one of $v$ and $\alpha$ is nonzero. If $v \neq 0$, then $\frac{1}{h^{3}} v^{T} \Phi^{T} \Phi v>0$, and consequently $x^{T} H x>0$. If $v=0, \alpha \neq 0$ we get

$$
x^{T} H x=\alpha^{2} v_{0}^{T} \mathbf{F} v_{0}>0 .
$$

In the next section, the general cross validation method is reviewed and a specific $G C V$ function is proposed for the $Q P(\mathbb{E . 8})$, that estimates the optimal value of the smoothing parameter $\varepsilon$.

\section{E.4 Generalized Cross-Validation}

The general cross validation method for smoothing splines was first developed by Wahba et al. in for instance [12], [13]. Here, a review is provided for general cross validation as proposed by [12], where the smoothing parameter is estimated for a problem similar to Problem E.2.1. Subsequently we derive an estimate of the general cross validation function for Problem E.2.1

\section{E.4.1 Background}

In [12], [13] Wahba et al. study a smoothing spline problem with solution $g_{N, \lambda}(t)$, defined by

$$
g_{N, \lambda}(t) \triangleq \underset{g: g^{(n)}(t) \in L_{2}}{\arg \min } \lambda \int_{0}^{1}\left(g^{(n)}(t)\right)^{2} d t+\frac{1}{N} \sum_{i=1}^{N}\left(g\left(t_{i}\right)-z_{i}\right)^{2},
$$

where $g^{(n)}$ is the $n^{\text {th }}$ derivative of $g(t)$ and $\left(t_{i}, z_{i}\right)$ are noisy samples of an underlying function $g_{\text {true }}(t)$. $g_{N, \lambda}(t)$ is a linear function of the data $z_{i}$. In particular, this means that

$$
\left[g_{N, \lambda}\left(t_{1}\right), \ldots, g_{N, \lambda}\left(t_{N}\right)\right]^{T}=S(\lambda)\left[z_{1}, \ldots, z_{N}\right]^{T}
$$

for a unique matrix $S(\lambda)$, called the influence matrix. $S_{i, j}(\lambda)$ is a measure of how much the measurement $z_{j}$ influences the spline $g_{N, \lambda}$ at $t_{i}$.

Ideally, the smoothing parameter $\lambda$ should be chosen to minimize the average square error, which is defined by

$$
R(\lambda) \triangleq \frac{1}{N} \sum_{i=1}^{N}\left(g_{N, \lambda}\left(t_{i}\right)-g_{\text {true }}\left(t_{i}\right)\right)^{2}
$$


However, minimizing $R(\lambda)$ requires knowledge of $g_{\text {true }}(t)$, at least at the measurement points. In [12] an estimate $\lambda_{G C V}$ of the minimizer of E.16 is found by minimizing the generalized cross calidation function $V_{G C V}(\lambda)$. Denote by $g_{N, \lambda}^{[k]}(t)$ the smoothing spline obtained when removing the $k^{\text {th }}$ data point prior to minimizing the cost function (E.14). Then $V_{G C V}(\lambda)$ is defined by

$$
\begin{aligned}
& V_{G C V}(\lambda) \triangleq \frac{1}{N} \sum_{k=1}^{N} \omega_{k}(\lambda)\left(g_{N, \lambda}^{[k]}\left(t_{k}\right)-z_{k}\right)^{2}=\frac{\frac{1}{N}\|(I-S(\lambda)) z\|^{2}}{\left(\frac{1}{N} \operatorname{trace}(I-S(\lambda))\right)^{2}}, \\
& \text { for } \omega_{k}(\lambda) \triangleq\left(\frac{1-s_{k k}(\lambda)}{\frac{1}{N} \operatorname{trace}(I-S(\lambda))}\right)^{2},
\end{aligned}
$$

where term $k$ in the sum is a measure of how well the spline $g_{N, \lambda}^{[k]}(t)$ predicts the data point $z_{k}$, and $\omega_{k}(\lambda)$ is a weight that compensates for unequal spacing of the data. It is shown in [12] that $\lambda_{G C V}=\arg \min _{\lambda} V_{G C V}(\lambda)$ has the following appealing property:

$$
\lim _{N \rightarrow \infty} \frac{E\left(R\left(\lambda_{G C V}\right)\right)}{\min _{\lambda} E(R(\lambda))}=1,
$$

where $E(\cdot)$ is the expectation value of $(\cdot)$. In other words, the expected mean square error using $\lambda_{G C V}$ tends to the minimum possible expected mean square error as $N \rightarrow \infty$.

In the next section, an estimate of the influence matrix for Problem E.2.1 is derived.

\section{E.4.2 GCV for Periodic Control Theoretic Smoothing Splines}

In this section, we derive a $G C V$ cost function for periodic control theoretic smoothing splines, based on an estimate $\hat{S}(\varepsilon)$ of the influence matrix for Problem E.2.1. This constitutes the main result of the paper.

The estimate $\hat{S}(\varepsilon)$ is computed from the discretization reviewed in Section E.3 and takes into consideration the constraints E.2.

This paper follows the convention used in [9-11]. To clarify, the relation between the smoothing parameters in E.1 and E.14 is $\varepsilon^{2}=1 / \lambda . \hat{S}(\varepsilon)$ should satisfy

$$
\left[r_{N, \varepsilon}\left(t_{1}\right), \ldots, r_{N, \varepsilon}\left(t_{N}\right)\right]^{T}=\hat{S}(\varepsilon)\left[z_{1}, \ldots, z_{N}\right]^{T},
$$

where $r_{N, \varepsilon}(t)$ is the optimal solution to Problem E.2.1 given the data set $\left(t_{i}, z_{i}\right)$ and the smoothing parameter $\varepsilon$. Recall that the vectors $\hat{t}, \hat{r} \in \mathbb{R}^{M}$ constitute the discretization of the spline $r(t)$. Let $\hat{r}_{t_{i}}$ denote the element of $\hat{r}$ corresponding to $r\left(t_{i}\right)$. The matrix $F$ defined by (E.6) "picks out" those elements of $\hat{r}$ :

Equation E.9 yields

$$
\left[\begin{array}{c}
\hat{r}_{N, \varepsilon, t_{1}} \\
\vdots \\
\hat{r}_{N, \varepsilon, t_{N}}
\end{array}\right]=F^{T}\left[\begin{array}{c}
\hat{r}_{N, \varepsilon, \hat{t}_{1}} \\
\vdots \\
\hat{r}_{N, \varepsilon, \hat{t}_{M}}
\end{array}\right]=F^{T} \hat{r} .
$$

$$
\hat{r}_{N, \varepsilon}=-H^{-1} c=\frac{\varepsilon^{2}}{N} H^{-1} F z=\frac{\varepsilon^{2}}{N}\left(\frac{1}{h^{3}} \Phi^{T} \Phi+\frac{\varepsilon^{2}}{N} F F^{T}\right)^{-1} F z .
$$

Therefore, we define

$$
\begin{aligned}
& \hat{S}(\varepsilon) \triangleq \frac{\varepsilon^{2}}{N} F^{T}\left(\frac{1}{h^{3}} \Phi^{T} \Phi+\frac{\varepsilon^{2}}{N} F F^{T}\right)^{-1} F \\
& \hat{V}_{G C V}(\varepsilon) \triangleq \triangleq \frac{1}{N}\|(I-\hat{S}(\varepsilon)) z\|^{2} \\
&\left(\frac{1}{N} \operatorname{trace}(I-\hat{S}(\varepsilon))\right)^{2}
\end{aligned}
$$


[18] states that $S(\lambda)$ is symmetric and positive semidefinite. It is straightforward to show that the estimate $[\mathrm{E.23}$ ) retains these properties.

\section{E.5 Simulations}

In this section, simulation results are provided to demonstrate properties of the proposed $G C V$ method. First, an example is shown to illustrate the usefulness and performance of the $G C V$ method. Then we investigate whether the asymptotic result (E.19) holds for the estimate $\varepsilon_{G C V}$. Throughout this section, the following notation is used:

$$
\begin{aligned}
& \varepsilon_{G C V}=\underset{\varepsilon}{\arg \min } \hat{V}_{G C V}(\varepsilon) \\
& \varepsilon_{\text {min }}=\underset{\varepsilon}{\arg \min } E(R(\varepsilon)), \quad \text { with } R(\varepsilon) \triangleq \frac{1}{N} \sum_{i=1}^{N}\left(r_{N, \varepsilon}\left(t_{i}\right)-r_{\text {true }}\left(t_{i}\right)\right)^{2} \\
& r_{N, \varepsilon}=\text { the spline computed using }(N, \varepsilon) \\
& r_{\text {true }}=\text { the underlying contour. }
\end{aligned}
$$

In simulation, the feasible region for $\varepsilon$ was restricted to the interval $\Delta \varepsilon=[1,1000]$. Deviations of $\varepsilon_{G C V}$ from $\varepsilon_{\text {min }}$ were computed as $\left\|\varepsilon_{G C V}-\varepsilon_{\text {min }}\right\| / \Delta \varepsilon$.

\section{E.5.1 Importance of Choice of Smoothing}

Here, an example is provided to demonstrate advantages of optimal smoothing. With an added noise $\sigma=0.1$ mean $\left(r_{\text {true }}\right)$ and using $N=100, M=800$, splines are generated with $\varepsilon=\varepsilon_{\text {min }}$, $\varepsilon=\varepsilon_{G C V}, \varepsilon=10 \varepsilon_{\min }$ and $\varepsilon=0.1 \varepsilon_{\min }$ to compare results for different values of $\varepsilon$. The cost functions $R(\varepsilon)$ and $\hat{V}_{G C V}(\varepsilon)$ are shown in Figure 1 and the resulting splines are shown in Figure 2 The advantages of optimal smoothing are clear from the figures. Due to space limitations, only one example contour is included. More examples are available at www.math.kth.se/ karasalo/GCV.pdf. In total, simulations were run for 25 test cases, with $\sigma, N$ and $M$ as above and the mean deviation of $\varepsilon_{G C V}$ from $\varepsilon_{\min }$ was less than $7 \%$.

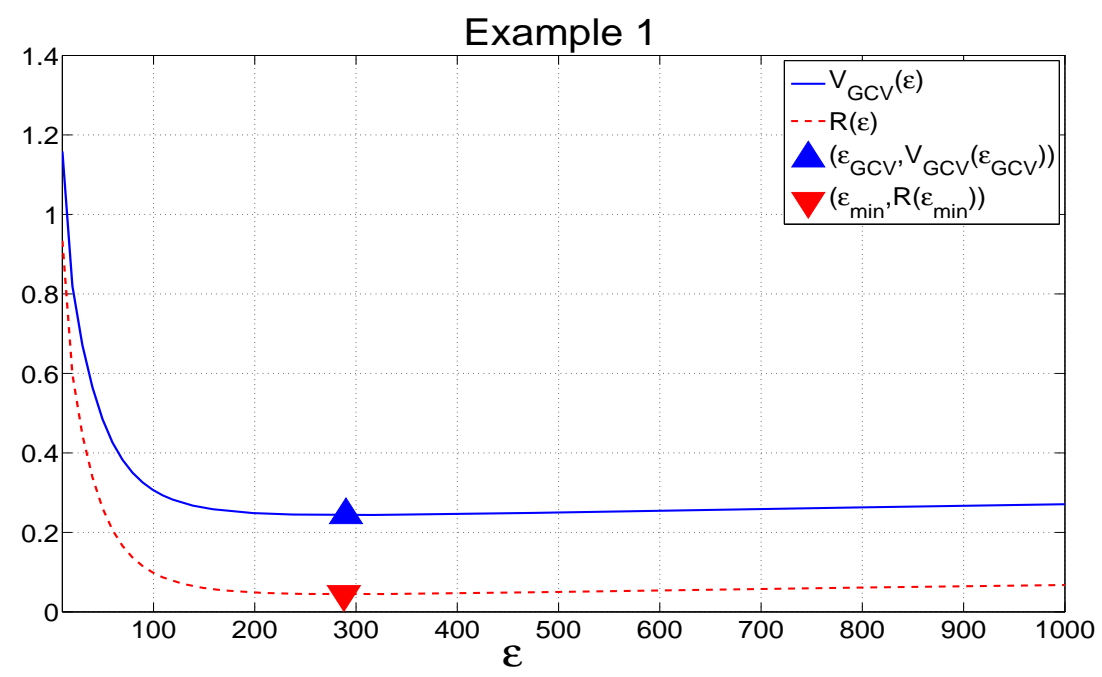

Figure 1: Importance of Choice of Smoothing: Cost functions for the example in Figure2. 


\section{E.5.2 Asymptotic properties}

In this section we investigate whether the asymptotic result E.19) holds for the estimate $\varepsilon_{G C V}$, i.e. if

$$
\lim _{N \rightarrow \infty} \frac{E\left(R\left(\varepsilon_{G C V}\right)\right)}{\min _{\varepsilon} E(R(\varepsilon))}=1 .
$$

This asymptotic optimality may be regarded as the most important property of the smoothing parameter. We have performed simulations for $M=1000$ and $N=\{1,2, \ldots, 1000\}$ for 25 arbitrary contours and a noise level of $\sigma=0.1$ mean $\left(r_{\text {true }}\right)$. Results are provided in Figure 3. We show mean values of $\varepsilon_{G C V}, \varepsilon_{\min }, R\left(\varepsilon_{G C V}\right)$ and $R\left(\varepsilon_{\text {min }}\right)$ for the 25 contours. $\varepsilon_{G C V}$ was generally a fair estimate of $\varepsilon_{\min }$, with a mean deviation of about $11 \% . R\left(\varepsilon_{G C V}\right)$ stays close to $R\left(\varepsilon_{\min }\right)$ and shows a clear decrease as $N$ increases. Finally, resulting error quotients are shown for increasing $N . R\left(\varepsilon_{G C V}\right) / \min _{\varepsilon} R(\varepsilon)$ is decreasing toward 1 , as expected.

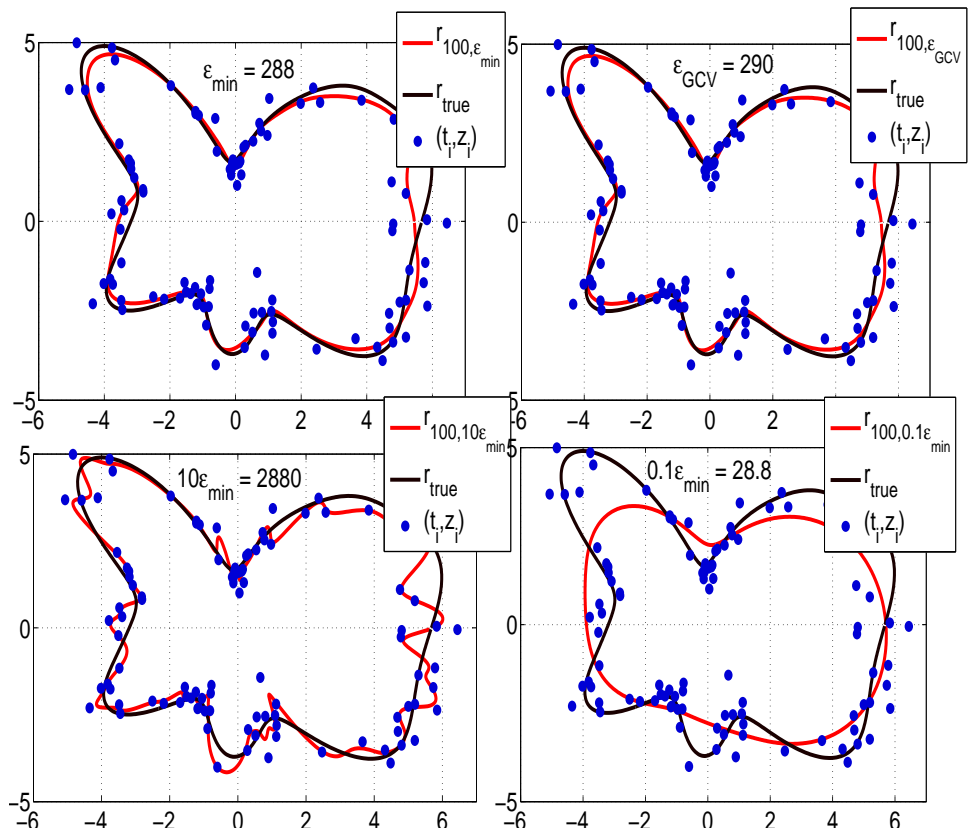

Figure 2: Importance of Choice of Smoothing: Splines generated with different values of $\varepsilon$. Top left: $\varepsilon=\varepsilon_{\text {min }}$. Top right: $\varepsilon=\varepsilon_{G C V}$. Bottom left: $\varepsilon=10 \varepsilon_{\text {min }}$. Bottom right: $\varepsilon=0.1 \varepsilon_{\text {min }}$.
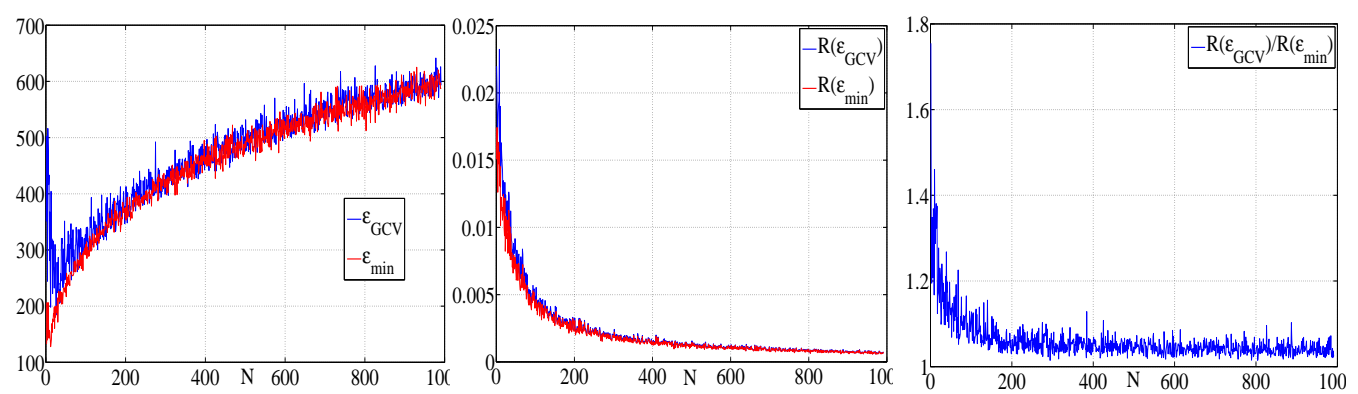

Figure 3: Asymptotic Properties: Mean values of over 10 test contours as $N \rightarrow M$. Left: Mean values of $\varepsilon$. Middle: Mean values $R\left(\varepsilon_{G C V}\right)$ and $R\left(\varepsilon_{\text {min }}\right)$. Right: $R\left(\varepsilon_{G C V}\right) / \min _{\varepsilon} R(\varepsilon)$. Mean values over 10 test cases for $N \rightarrow M$. 


\section{E.6 Conclusions}

In this paper, a general cross validation function was derived based on a discretization of a periodic control theoretic smoothing spline problem. An estimate of the optimal smoothing parameter $\varepsilon$ was found by minimizing a $G C V$ cost function $\hat{V}_{G C V}(\varepsilon)$, without a priori information about the underlying closed curve or the quality of data. Theoretical and simulation results regarding properties of $\hat{V}_{G C V}(\varepsilon)$ and the corresponding influence matrix $\hat{S}(\varepsilon)$ were provided and an example was shown to illustrate the usefulness and performance of the method.

\section{E.7 References}

[1] C.F. Martin and J. Smith, Approximation, interpolation and sampling, Differential geometry, the interface between pure and applied mathematics, Contemporary Mathematics, vol. 68, pp. 227-252, 1987.

[2] C.F. Martin, S. Sun, and M. Egerstedt, Control theoretic smoothing splines, IEEE Transactions on Automatic Control, vol. 45, pp. 2271-2279, 2000.

[3] M. Egerstedt, C.F. Martin, and S. Sun, Optimal control, statistics and path planning, Mathematical and Computer Modelling, vol. 33, pp. 237-253, 2001.

[4] M. Egerstedt and C.F. Martin, Statistical estimates for generalized splines, ESAIM:Control, Optimisation and Calculus of Variations, vol. 9, pp. 553-562, 2003.

[5] Y. Zhou, W. Dayawansa, and C.F. Martin, Control theoretic smoothing splines are approximate linear filters, Comm. in Information and Systems, vol. 4, pp. 253-272, 2004.

[6] H. Kano, H. Fujioka, M. Egerstedt, and C.F Martin, Optimal smoothing spline curves and contour synthesis, Proc. of the 16th IFAC World Congress, 2005.

[7] H. Kano, M. Egerstedt, H. Fujioka, S. Takahashi, and C.F. Martin, Periodic Smoothing Splines, Automatica, vol. 44, pp. 185-192, 2008.

[8] M. Egerstedt and C.F. Martin, Control Theoretic Splines: Optimal control, statistics and path planning, Princeton Series in Applied Mathematics, to appear 2010.

[9] M. Karasalo, X. Hu, and C.F. Martin, Localization and Mapping using Recursive Smoothing Splines, Proc. of the European Control Conference (ECC), 2007.

[10] M. Karasalo, X. Hu, and C.F. Martin, Contour Reconstruction and Matching using Recursive Smoothing Splines, Modeling, Estimation and Control, Springer, pp. 193-206, 2007.

[11] M. Karasalo, G. Piccolo, D. Kragic, and X. Hu, Contour reconstruction using recursive smoothing splines - Algorithms and experimental validation, Robotics and Autonomous Systems, no. 57, pp. 617-628, 2009.

[12] P. Craven and G. Wahba, Smoothing Noisy Data With Spline Functions, Numerische Mathematik, vol. 31, pp. 377-403, 1979.

[13] G. Wahba, Spline Models for Observational Data, CBMS-NSF Series, SIAM, 1990.

[14] R.L. Eubank, Nonparametric Regression and Spline Smoothing, 2nd ed., CRC Press, 1999.

[15] C.H. Reinsch, Smoothing by spline functions, Numerische Mathematik, vol. 10, pp. 177-183, 1967. 
[16] M.F. Hutchinson and F.R. de Hoog, Smoothing Noisy Data with Spline Functions, Numerische Mathematik, vol. 47, pp. 99-106, 1985.

[17] M.F. Hutchinson and F.R. de Hoog, An Efficient Method for Calculating Smoothing Splines using Orthogonal transformations, Numerische Mathematik, vol. 50, pp. 311-319, 1987.

[18] M.F. Hutchinson, A stochastic estimator of the trace of the influence matrix for laplacian smoothing splines, Communications in Statistics - Simulation and Computation, vol. 19, no. 2, pp. 433-450, 1990.

[19] L. Eldén, A note on the computation of the generalized cross-validation function for illconditioned least squares problems, BIT Numerical Mathematics, Springer Netherlands vol. 24, no 4, 1984.

[20] B. Shahraray and D.J. Anderson, Optimal estimation of contour properties by cross-validated regularization, IEEE Trans. on Pattern Analysis and Machine Intelligence, vol. 11, no. 6, 1989. 